\title{
32
}

\section{Computer based teaching and learning in statistics}

\author{
Noel Wilson \\ Sally McClean \\ University of Ulster \\ Newtownabbey \\ Northern Ireland
}

\begin{abstract}
This paper describes work associated with an Information Technology Training Initiative (ITTI) Project which is delivering a range of products in support of basic statistics and quantitative methods training. We describe the project philosophy and the background to it, the product design criteria and their development, current and projected usage, spin-off activity, evaluation and usability studies, and future plans. The project deliverables have already demonstrated their benefits as a learning resource within IT Training Workshops, as materials suitable for a final-year degree module and as selfstudy materials. Uptake of products by other universities will be briefly referred to and also their relevance to teaching, learning and training situations.
\end{abstract}

Main conference themes: flexible learning

Educational areas: industry training

Study topics: mathematics

Secondary keywords: courseware, learning materials, teaching materials 


\section{INTRODUCTION}

This project, an Information Technology Training Initiative (ITTI) Project and one of 23 funded by the then Universities Funding Council (UFC) Information Systems Committee (ISC) was established in August 1991 with the award of $£ 69,462$ being made to the university by the Department of Education for Northern Ireland (DENI). An additional grant of $£ 7,800$, matched by consultancy, software and training from SAS (UK) Ltd., enabled us to extend the project's schedule of deliverables to include products in support of the SAS software system. Our commitment was to the production of a range of training products which would help Computer Centre staff support their customers with the use of standard statistical packages and associated activities (e.g. questionnaire design). With the exception of one product, a workbook on Questionnaire Design, the emphasis would be on the use of Computer Based Tutorials (CBTs) for information delivery as we envisaged the products being used in open learning centres managed by Computer Centres and Staff Development Departments. The schedule of deliverables consists of 9 CBTs, covering the use of selected statistical procedures within Lisrel $^{\circledast}$, MINITAB $^{\circledast}$, SAS $^{\oplus}$ and SPSS ${ }^{\circledR}$, with accompanying documentation and example datasets. Topic coverage is broad and representative of that encountered in many tertiary-level courses where statistics or quantitative methods is taught as a secondary subject.

Whilst the original thrust of ITTI was focused on training it has been accepted that many of the projects are creating resources which are relevant to teaching and learning situations, especially now that the UK university sector is experiencing a significant growth in its student population and also because of the emerging interests in student centred learning experiences. Within our university we are using our own CBTs in support of Information Technology (IT) training Workshops as open learning resources on Local Area Networks (LANs). And also as directed learning materials in a final year degree module and as exemplars for computing science and other students who undertake project and dissertation work involving CBT production. We have also benefited from other projects within ITTI; acquisitions have facilitated new areas of IT training and enhanced the delivery of others. These experiences are addressed later in this paper.

\section{Project background}

The University of Ulster was established in 1984 by the merging of the New University of Ulster and the Ulster Polytechnic. The former institutions had their respective strengths in research and teaching. In the 1980s research was still considered the focus of university activity so the new institution's Research 
Committee established a Working Group (WG) to examine how it could develop research skills in those staff (principally from within the former Polytechnic sector) whose expertise was principally in consultancy and teaching. The WG recommended the establishment of a module based Research Methods programme to deliver a series of topics relevant to the needs of its target audience. This recommendation was accepted and the new course established with internal and external validation procedures comparable to those adopted for primary and postgraduate degree courses. Several modules of the Research Modules course focused on computer based skills for social and behavioural science research and were applicable to staff in the fields of business and management education, social and health sciences, and education. Demand for the course was high in its first year: the full range of modules being run twice, once during the academic year and once during the summer vacation period. In subsequent years there was a steady drop off in demand for some modules as staff needs were met. There has also been a need to develop new modules to satisfy emerging needs and also to offer particular modules on a demand basis.

The announcement of ITTI provided an ideal opportunity to seek funding to enable a more flexible approach to course delivery to be adopted. The basis of our proposal was the packaging of all the statistical modules to provide learner centred products. Choice of the statistical modules was deliberate as the Invitation for Proposals communication clearly stated that 'proposals should address areas which would enable Computer Centre staff support users in a more effective way'. The University Computer Centre, like many others, provides a user support service which handles queries in the areas of survey analysis, questionnaire design and use of statistical packages amongst other topics. Our proposal was a joint submission by Computer Centre and School of Information and Software Engineering, as colleagues from both areas taught on the Research Methods modules [1, 2].

\section{THE PROJECT}

\section{Topic coverage}

Topics typical to those encountered in a broad range of statistics/quantitative methods courses were selected. The topics are applicable to those reading for higher degrees, undertaking consultancy and to primary and postgraduate degree students in areas where statistical techniques are secondary to the subject of investigation. 
Topics addressed include:

- nature of data, concept of measurement, statistical variables;

- aspects of sampling, questionnaire design, data collection and cleaning;

- presentation graphics: bar and pie charts, histograms, scatterplots and stemand-leaf plots;

- tabulating data: one- and two-way tabulations including counts percentages;

- summary measures of location and spread: mean, median, mode and standard deviation, variance;

- measures of association: Chi-squared statistic and Pearson's Coefficient;

- statistical inference: techniques for 1,2 and $>2$ samples of data measurements on nominal, ordinal and interval scales;

- simple regression analysis;

- scale construction and concept of reliability;

- selected multivariate techniques: multiple regression, factor analysis and principal component analysis.

\section{Software coverage}

Throughout the United Kingdom (UK) tertiary education system, and indeed worldwide, Minitab and SPSS have established themselves as leaders in the field of general statistical analysis and these software tools therefore feature heavily in our products. We also include SAS and Lisrel; SAS because it is another well known analysis tool, albeit somewhat less popular as a general tool in the UK, and Lisrel because of its popularity in social science and psychology teaching and research applications. Most CBTs offer the user a balance between theory and practice with reference to a proprietary package being made to illustrate the conduct of a particular analysis.

\section{Mode of presentation}

Generally speaking, the target audience for our products do not have a strong motivation for computer usage nor for the subject area. Many potential users will not have studied mathematically orientated subjects beyond secondary level education and do not wish to be confronted with a theoretical approach to data analysis. However, it is nevertheless important that they be presented with a rigorous, yet nonmathematical, treatment of the topics, otherwise an appreciation of applied statistics will not be acquired. Our philosophy is to introduce a minimal amount of theoretical background and to do this in a highly intuitive manner supplemented as appropriate with carefully chosen illustrative examples. This philosophy applies equally to the CBT and its companion paper based materials (collectively referred to as courseware). 


\section{CBT design philosophy}

Production of computer software is an exacting and time consuming task. Conscious of the fact that our products would relate to proprietary products, there was a requirement to consider the concept of product shelf life or the long(er) term viability of our courseware. The idea of developing consumable type products was considered to be the most cost effective solution as it was impossible to anticipate the look-and-feel of future releases of the proprietary statistical packages. Furthermore, to adopt software design principles which would futureproof our initial investments would have been prohibitively expensive and disruptive to delivery schedules. The Project Team agreed on a 12 week development time for each CBT, on a modular design approach and on reusable code. This approach has been applied successfully to a number of products.

Human-computer interaction has also been a major consideration given our emphasis on learner centred learning. The need to design an interface which is intuitive and simple, yet attractive and attention focusing, was important. Each product has been designed in a consistent manner employing the idea of a hypertext for subject organization with a simple control panel at the base of each screen to enable courseware navigation and a module name displayed at the top of screen to identify location within the hypertext. With the exception of two CBTs for SAS the Guide hypertext system adopted by several of the ITTI Project holders was the development language.

Users are presented with several features consistent with the notion of a learner centred tool, these include:

- practice sessions for gaining experience in the use of the statistical packages;

- short exercises at end of major sections, usually of yes/no or true/false type;

- multiple choice question sets for subject consolidation;

- scoring to provide a basic indication of topic comprehension.

Each CBT is accompanied with a Workbook which includes additional exercises designed to be undertaken using the skills and knowledge acquired through use of the tutorial. Many of these exercises may require the user to work directly with the proprietary software.

\section{IMPACT OF PROJECT}

\section{Experiences within research methods course}

Part of our original project proposal dealt with product testing and implementation: our approach was to plan product prerelease dates so that these could be trailed within Research Methods course modules for which they were 
being developed. This approach proved to be successful. The Introductory Statistics using Minitab product has been introduced to the Descriptive Statistics module. Past participants on this, and other modules requested additional time for hands-on experience. Module redesign to facilitate use of the CBT enabled this request to be addressed. Responses from the cohort were positive and the module tutor was able to allocate more time to dealing with individual's particular difficulties. It was also observed that participants were able to proceed at their own pace thus affording the more able to undertake additional examples and the less able to gain confidence and experience through extended interaction with the CBT. A short evaluation survey was conducted and all respondents reported positive experiences.

The two CBTs which were introduced for the SAS statistical package coincided with the introduction of the SAS software service to the university. Our Research Methods course was extended to include a new module on SAS usage ( 2 days) with the design of the module being centred on the use of the CBTs as the principal delivery vehicle. As referred to already the desire of participants for extensive hands-on experience was again addressed. An additional Workbook of Examples was developed to accompany the materials for the ITTI products. Delivery of this module required minimal lecture style input from the course tutor who now assumed a role of facilitator as opposed to tutor. Evaluation of the delivery of this module was by completion of Module Evaluation Questionnaires and informal observation. Only 1 (of 9) participants reacted negatively to their experience (and this was found to be because of computer related difficulties not associated with the CBTs). Overall the coverage of product features, examples and topics within this course was greater than what could have been achieved through traditional workshop style course delivery methods.

Both of these evaluations are associated with the use of the products in a context which blends training, teaching and learner centred learning activities.

\section{Experiences on other courses}

Despite the aim of the project being the provision of resources for a target audience studying or using statistics as a secondary subject we have used our SAS CBTs within an assessed final-year module of our BSc degree in Mathematics, Statistics and Computing course. SAS experience is in high demand especially within the pharmaceutical industry and is therefore relevant to this course. With the impact of modularization and the need to retain a significant portion of formally taught materials a learner centred approach to the introduction of SAS was adopted (Some students gain hands-on experience through their industrial placement year activities). A class of 25 students was given an assignment which necessitated them using the CBTs from the open 
learning microcomputer laboratories and then writing a Report on their experiences. Since the group was also taking computing modules a product usability study was also incorporated thus exposing them to a commercial product [3] which was relevant to their studies in the fields of human-computer interaction and software engineering.

Results of the usability study [4] classified the products as above average and indicated that our design goals in the areas of software learnability, helpfulness, control and user perceived quality had all been met. Indeed, students who encountered SAS during their placement year commented that they could have benefited from an introduction to the tutorials at an earlier date. This mode of use of SAS CBTs has occurred in two successive years.

Further detailed evaluation work has been undertaken using our Reliability and Scale Construction CBT. It has been evaluated by a group of experienced staff and research students who attended a one week residential course on Multivariate Statistics. They were all experienced computer users and also experienced in the application of statistical methods. Feedback on the usefulness of the CBT as a learning aid was positive. Several of the group $(\mathrm{N}=20)$ indicated the advantages of CBTs as an independent learning aid both for themselves and, in instances where the respondent was a lecturer, for their students.

SUMI was also used in the evaluation of the Reliability and Scale Construction CBT. A Year IV undergraduate Psychology group $(\mathrm{N}=18)$ was introduced to the product as a revision aid for the topics being covered in Year II of their course. The experiment required the completion of a questionnaire, which solicited opinion on the product content and layout in addition to its suitability as a learning resource. SUMI results rated the usability of the CBT as above average with the helpfulness score being highest (median $=62$ against a normative value of 55 for reasonably acceptable commercial software). Questionnaire results on a set of Likert-scale questions consistently manifest the perceived benefits of a CBT when compared to conventional teaching methods and resources.

A Year III undergraduate Psychology group $(\mathrm{N}=40)$ and an MSc in Applied Psychology group $(\mathrm{N}=8)$ have been using modules of the Reliability and Scale Construction CBT in a mixed delivery style of teaching. In both instances the class is introduced to aspects of the topic using conventional lectures and are then directed to use specific modules of the CBT to gain an appreciation of the analytic tools. This is followed by a computer practical which involves the students in hands-on analysis and interpretation of results. No formal evaluation has as yet been undertaken, however observation indicates that the teaching process is much more efficient and that there is benefit to be gained 
from using CBTs to explain software related processes and the associated computer generated results.

\section{Other uses of products}

Of particular relevance to this paper are the secondary benefits being gained from our CBT products. Use of hypertext and authoring based languages is popular in several computing courses at primary and postgraduate level. Student experience is gained through the conduct of an extensive project involving the production of software. Computer Centre staff is frequently called upon to provide consultancy for these students especially in the technical features of the software development systems. It is here that we have been able to make significant savings in time through providing students with the source code of our products for their own investigation. In this way the student is afforded an opportunity to learn new techniques and to experience at first hand the crafting of a production piece of software by experienced personnel. Their learning experience is enhanced in a way which would not be possible during the formal taught units of their course as the time required could not be allocated as either taught unit time or as assignment activity.

We have also supported students whose industrial placement year has been undertaken within the university. Two students have been involved in the production of selfstudy units on economics, a third has completed a module on discrete mathematics which is being used to displace formal teaching time in a foundation unit on software engineering. Another has developed several units on statistics for nurse education and another has produced an introductory level CBT for numerical methods for engineers. All of these initiatives have been triggered by the moves towards modularization and learner centred learning.

\section{External usage}

A condition of award of grant is that all products will be made freely available to the UK tertiary level community at a cost which reflects product packaging and distribution. Our products are being shared and have been taken up by several other institutions. For example, over 70 copies of our Introductory Statistics using Minitab product have been sold, over 40 of our SAS products and around 30 of our SPSS/PC+ Data Entry module. Approximately 50 prerelease copies of our Workbook on Questionnaire Design were distributed at an Exhibition of ITTI Products, over 200 were sold internally and over 800 to other Universities.

Each of our products contains a Product Evaluation Questionnaire which we hope will be completed and returned so as to facilitate further product evaluations and improvements. Often, via electronic mail, we can record that a product is being favourably received although it has not yet been possible to 
ascertain the extent to which these are being used at other sites. We are also interested in the mode of use and the number of students which is benefiting from the project.

\section{CONCLUSIONS}

This project has now reached its concluding stages with the final product scheduled for delivery in September 1995. The impact of the project deliverables on changing modes of course delivery has already been significant and this trend is likely to continue. The university has just approved an MRes degree (first intake in January 1995) which will increase usage of products as these are listed as a resource for the course.

We feel that the project has clearly demonstrated the benefits of computer based subject material delivery in 'liberating the learner' offering:

- a flexible approach to learning;

- opportunities for student centred assessment of progress;

- increased lecture time for dealing with other aspects of course activity;

- a freedom of choice and an opportunity for student motivation through gaining a personal sense of achievement.

\section{REFERENCES}

1. Wilson, R. N. and Mc Clean, S. I. (1993) Production of Computer-based Tutorials in support of Statistical Applications-A Case Study from Higher Education. Presented at SEUGI'93 Jersey, Channel Islands.

2. Mc Clean, S. I., Scotney, B. and Wilson, R. N. (1994) Computer-based Training for Statistical Applications. Presented at CASI'94 Dundalk, Ireland.

3. Human Factors Research Group, University of Cork Ireland. Software Usability Measurement Instrument (SUMI).

4. Mc Mullan, T. C. A. et al (1993) Usability Report on SAS Computer-based Training Products. Internal Report University of Ulster. 